\title{
Morphometric age and survival following kidney transplantation
}

\author{
Michael N. Terjimanian ${ }^{1}$ (D) | Patrick W. Underwood ${ }^{1}$ | David C. Cron ${ }^{1}$ (D) | \\ Joshua J. Augustine ${ }^{2}$ | Kelly A. Noon ${ }^{2}$ | Devan A. Cote ${ }^{2}$ | Stewart C. Wang ${ }^{1}$ | \\ Michael J. Englesbe ${ }^{1}$ | Kenneth J. Woodside ${ }^{1}$
}

${ }^{1}$ Department of Surgery, Morphomics Analysis Group (MAG), University of Michigan, Ann Arbor, MI, USA

${ }^{2}$ Case Western Reserve University \& University Hospitals, Cleveland, OH, USA

\section{Correspondence}

Michael N. Terjimanian, MSE, University of Michigan Medical School, Ann Arbor, MI, USA. Email: terjiman@med.umich.edu

\begin{abstract}
Background: As patients with chronic kidney disease become older, there is greater need to identify who will most benefit from kidney transplantation. Analytic morphomics has emerged as an objective risk assessment tool distinct from chronologic age. We hypothesize that morphometric age is a significant predictor of survival following transplantation.

Methods: A retrospective cohort of 158 kidney transplant patients from 2005 to 2014 with 1-year preoperative imaging was identified. Based on a control population comprising of trauma patients and kidney donors, morphometric age was calculated using the validated characteristics of psoas area, psoas density, and abdominal aortic calcification. The primary outcome was post-transplant survival.

Results: Cox regression showed morphometric age was a significant predictor of survival (hazard ratio, 1.06 per morphometric year [95\% confidence interval, 1.03-1.08]; $P<.001$ ). Chronological age was not significant (hazard ratio, 1.03 per year [0.98-1.07]; $P=.22$ ). Among the chronologically oldest patients, those with younger morphometric age had greater survival rates compared to those with older morphometric age.

Conclusions: Morphometric age predicts survival following kidney transplantation. Particularly for older patients, it offers improved risk stratification compared to chronologic age. Morphomics may improve the transplant selection process and provide a greater assessment of prospective survival benefits.
\end{abstract}

KEYWORDS

clinical decision-making, patient survival, risk assessment, risk stratification

\section{1 | INTRODUCTION}

The determination of which patients are suitable for transplant candidacy has always been a challenging task for clinicians. Transplant committees often rely on subjective physician assessments, collectively referred to as the "eyeball test," to formulate a patient's perioperative risk and long-term term survival. ${ }^{1-4}$ Patient age is one of the most critical factors of this assessment, but age itself can be a poor proxy for physiologic reserve-especially within the context of chronic kidney disease (CKD) and end-stage renal disease (ESRD), as well as other debilitating comorbidities. ${ }^{5-9}$ As the CKD population becomes older, better objective measures of preoperative risk are needed to distinguish older patients with potentially greater survival benefit than predicted based on chronological age alone.

Recently, our group has attempted to objectively measure this assessment using morphometric measurements found on crosssectional imaging in different types of surgical patients. ${ }^{10-12}$ By standardizing the baseline morphometric characteristics of aging in the 
general population, namely living kidney donors and trauma patients, a morphometric age was quantified to reflect these changes and found to be a distinct surgical risk factor from chronologic age. Thus far, morphometric age has been shown to correlate to outcomes in both the general surgical and liver transplantation populations. ${ }^{13,14}$ We aim to apply these findings to the kidney transplant recipients. Many transplant centers obtain abdominopelvic or pelvic computed tomography (CT) scans already for various indications, but most frequently for assessment of vascular targets for kidney engraftment. These scans have additional information that can be brought out with morphomic analysis, suggesting that this approach could have minimal cost impact. With increased focus on clinical outcomes and resource utilization, optimizing patient and graft outcomes, coupled with avoiding futile transplants, is vital. ${ }^{15}$

As the population of those with ESRD seeking renal transplant has become older, differentiating between the elderly patient with the physiological reserve to do reasonably well with the transplant operation and recovery, vs those too frail to do well, has become a progressively important focus for transplant centers. In this study, we sought to determine the relationship between morphometric age as an indicator of frailty and post-kidney transplant survival. We hypothesize that increased morphometric age will significantly correlate with poorer outcomes.

\section{2 | MATERIALS AND METHODS}

\section{1 | Study population}

This study was reviewed and approved by the Institutional Review Boards of both the University Hospitals Case Medical Center (UHCMC, IRB \#07-13-31) and the University of Michigan (UMHS, IRB \#HUM00041441). This study was a retrospective examination of patients who underwent kidney transplantation at UHCMC between January 2005 and May 2014, and who had a CT scan in the year prior to transplant. At UHCMC, abdominopelvic or pelvic CT scans were ordered annually (per clinical protocol) for kidney transplant candidates who met the following criteria: age $\geq 50$ years, diabetes, significant cardiovascular risk, or previous transplant. Scans were obtained in these patients for operative planning to assess for iliac artery calcifications and potential anastomotic targets. We also included CT scans ordered for other clinical indications, as long as they were within 1 year pre-transplant. In patients with multiple pretransplant CT scans, we selected the scan closest to transplant. All scans were noncontrast. We excluded patients who received a simultaneous pancreas or liver transplant. Typical postkidney transplant immunosuppression regimens included tacrolimus, which switched to cyclosporine if post-transplant glucose intolerance developed. All patients typically received methylprednisolone for 4 days post-transplant, without further maintenance steroids unless the patient was immunologically higher risk. Patients with delayed graft function generally remained on steroids.

\subsection{Analytic morphomics}

CT scans were processed using semi-automated algorithms programmed into MATLAB v13.0 (MathWorks; Natick, MA, USA). As described in our previous work, ${ }^{10-12}$ total psoas, psoas muscle density, and aortic wall calcification were measured after identifying individual vertebral levels on each patient's CT scan. Briefly, total psoas area was measured as the cross-sectional areas of the left and right psoas muscles at the level of L4. Using automated method, each pixel within the area encompassed was sampled for its radiologic density (in Hounsfield Units [HU]). Aortic calcification was measured as a percentage of the aortic wall surface area from L1 to L3. These measurements are shown in Figure 1. As previously described, ${ }^{13,14}$ morphometric age was computed as a function of the morphometric characteristics (psoas area, density, and aortic calcification) of a control population-namely potential kidney donors and
(A)

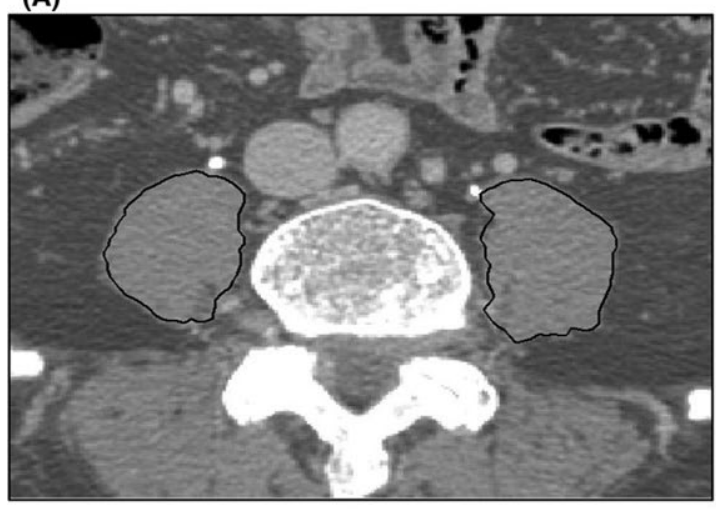

(B)

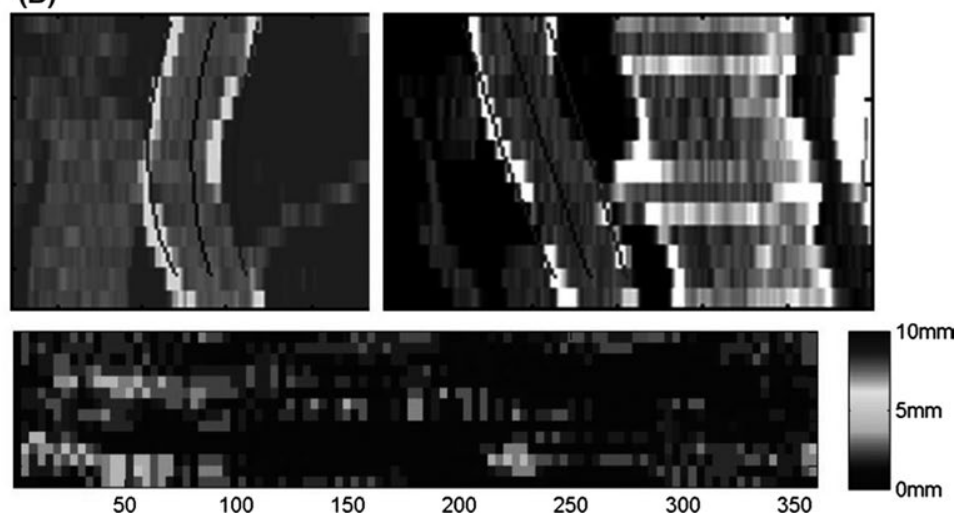

FIGURE 1 (A) Using semi-automated algorithms, the psoas muscles were outlined and total psoas area (TPA), and psoas density were determined. (B) Abdominal aortic calcification was measured on the wall of the infrarenal aorta from L1 to L3. After determining the centerline and radius, calcification was identified based on a relative threshold from the aortic lumen and expressed as a percentage of the total wall area. Below, these findings are further visualized in a two-dimensional mapping of the selected aortic wall. Individual pixels can be assessed for calcification severity by measuring the thickness of calcification (not utilized in this study) 
randomly selected trauma patients. The algorithm developed from this standardized population was applied to the study population to determine each patient's morphometric age.

\section{3 | Clinical data}

Clinical data were retrospectively collected from the electronic medical record at UHCMC. Data were de-identified, encoded, and sent to UMMS to be merged with the de-identified and coded morphomic data. Clinical variables collected included: age, sex, race, body mass index (BMI), chronic kidney disease (CKD) etiology (hypertension, diabetes mellitus, autoimmune, or other), hepatitis C (HCV) viral infection, waitlist duration, dialysis vintage pretransplant, prior kidney transplant, deceased donor, expanded criteria donor (ECD) status, donor age, prednisone post-transplant, calcineurin inhibitor use post-transplant, primary nonfunction, and delayed graft function.

\section{4 | Statistical analysis}

Descriptive statistics were computed for the study population. Continuous variables were summarized by mean and standard deviation, and frequency tables were produced for categorical variables. The Pearson product moment correlation, $r$, was used to assess collinearity between the two age metrics. Kaplan-Meier analysis was performed to stratify survival based on tertiles of chronologic and morphometric age. Standard survival analysis using Cox regression was performed to determine the covariate-adjusted effect of morphometric age on post-transplantation mortality. Patients began follow-up at the time of transplantation and continued until the earliest of death or loss to follow-up. Adjustment covariates include all variables listed above. All available covariates were initially evaluated univariately, and those with $P<.20$ were entered into the multivariate model. ${ }^{16,17} \mathrm{~A}$ final subset of adjustment criteria was determined using backwards selection. All analyses were performed using STATA

TAB LE 1 Patient demographic and clinical data

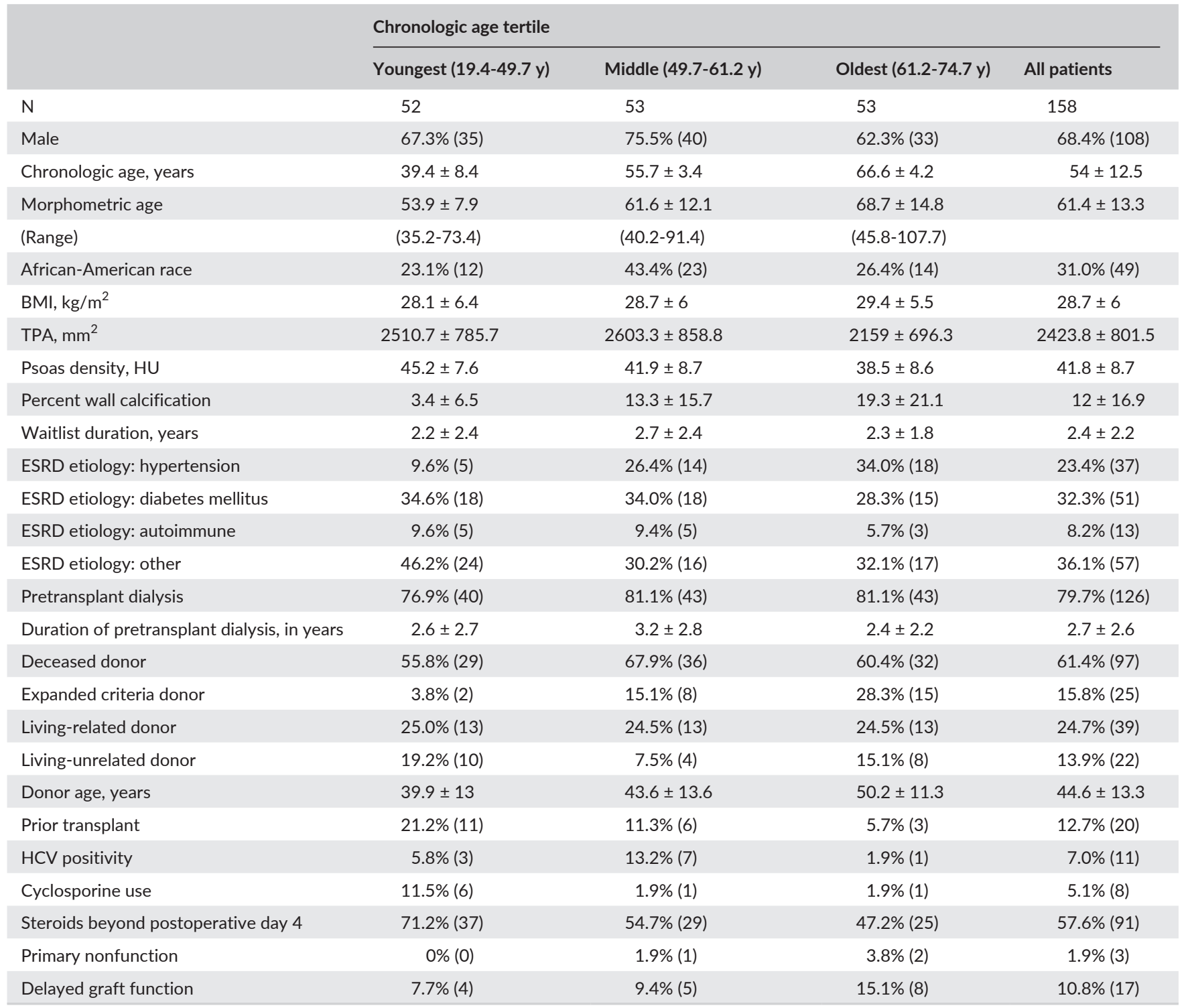

Continuous variables presented as mean \pm SD . 
v14.0 (College Station, TX, USA). A two-sided significance of $P<.05$ was used for all analyses.

\section{3 | RESULTS}

\section{1 | Study population}

Overall, 158 adult patients who underwent kidney transplantation received a 1-year preoperative CT scan, which included the regions of interest for morphometric assessment. These patients served as the study cohort. Demographics of the study population are shown in Table 1, stratified by tertiles of chronologic age. The mean chronologic age at transplant was $54.0 \pm 12.5$ years, and the mean morphometric age was $61.4 \pm 13.3$. Overall, $66.5 \%(n=105)$ of patients had a morphometric age greater than their chronologic age. Deceaseddonor kidneys were utilized in $61.4 \%(n=97)$ of cases, and living related donors for $24.7 \%(n=39)$. Overall, expanded criteria donors (ECD) accounted for $15.8 \%(n=25)$.

Further analysis shows that there is a positive correlation between chronologic and morphometric age, with $r=.49$. This correlation decreased as older patients are compared. Among the tertile of youngest chronologic age, $r=.34$; among the middle tertile, $r=.26$; among the oldest tertile of chronologic age, $r=.15$.

\section{2 | Graft survival}

Death-censored graft loss was observed in $10.8 \%$ (17) of patients. At 1 year after transplant, death-censored graft loss was seen in $1.9 \%$ (3) of patients. At 5 years, death-censored graft loss was seen in $7.6 \%$ (12) of patients. While these patients were both chronologically older (56.1 vs 53.7 years, $P=.55$ ) and morphometrically older (66.4 vs 60.8 years, $P=.13$ ) than those who did not experience graft loss, the differences were not significant when age was utilized as a continuous variable. When patients are stratified into tertiles of chronologic and morphometric age, the differences in graft loss among the tertiles also were not significant $(P=.72$ and $P=.92$, respectively).

Overall uncensored graft loss was observed in $28.5 \%(n=45)$ of patients, with $6.3 \%$ (10) occurring at 1 year and $20.3 \%$ (32) occurring at 5 years. When patients are stratified into tertiles of age, the differences in five-year uncensored graft loss were not significantly different $(P=.16)$ among the chronologic age tertiles, but were significantly different among morphometric age tertiles $(P=.008)$.

\section{3 | Patient survival}

Overall patient survival at 1 year, 3 years, and 5 years was $93.7 \%$ (148/158), 86.2\% (112/130), and 74.2\% (66/89), respectively. Cardiac etiologies were the most common cause of death, representing $50 \%$ of deaths at 1 year and $43.5 \%$ at 5 years. The remaining causes of death at 1 year were infections (30\%) and malignancy (10\%), with $10 \%$ of deaths unspecified. The remaining causes of death at 5 years were infections (17.4\%), malignancy (8.7\%), trauma (4.3\%), and suicide (4.3\%), with $21.7 \%$ of deaths unspecified.
Figure 2 shows Kaplan-Meier survival stratified by tertiles of chronologic age and morphometric age, respectively. In comparing tertiles of chronologic age, the youngest patients had much greater survival compared to the middle and oldest patients, as would be expected. Similarly, when comparing the tertiles of morphometric age, younger and middle age patients had much greater survival compared to the morphometric oldest patients. When the chronologically oldest patients are stratified by tertiles of morphometric age, as shown in Figure 3, the morphometric young and middle age tertiles had greater survival rates compared to the oldest. A similar finding was observed for the chronologic middle-aged tertile, while the chronologic youngest tertile did not show significant differences when stratified by morphometric age.
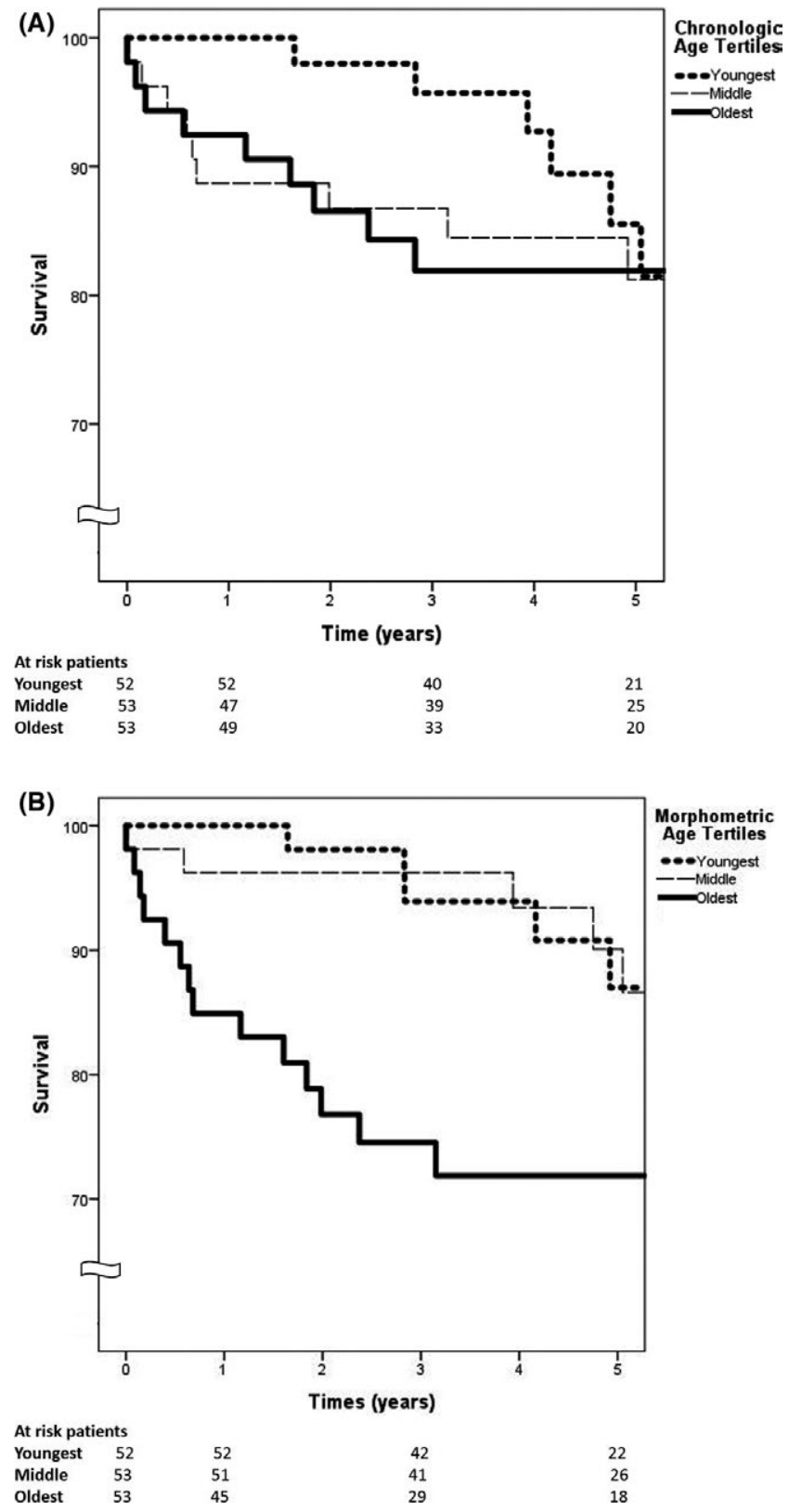

FIGURE 2 Kaplan-Meier survival curves were determined for the study population, stratified by both tertiles of chronologic age (A) and morphometric age $(B)$ 

(A) Youngest Chronologic Age Tertile

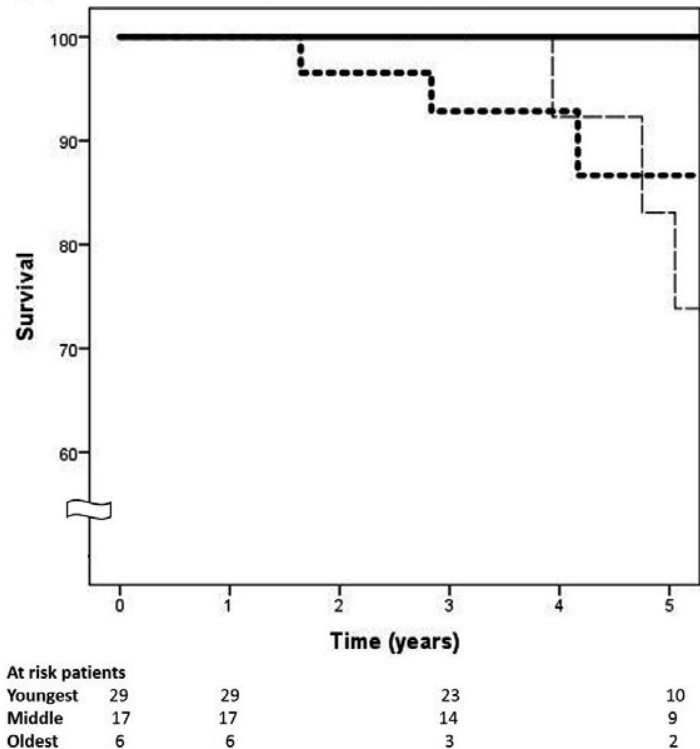

(C) Oldest Chronologic Age Tertile

Morphometric Age Tertiles - - Youngest - Middle - Oldest

(C)

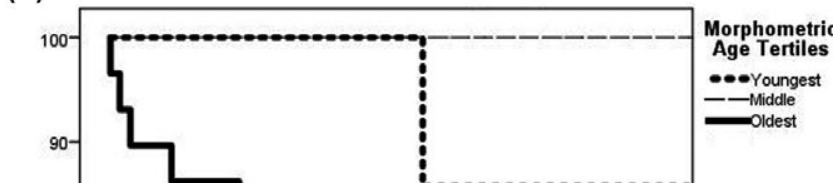

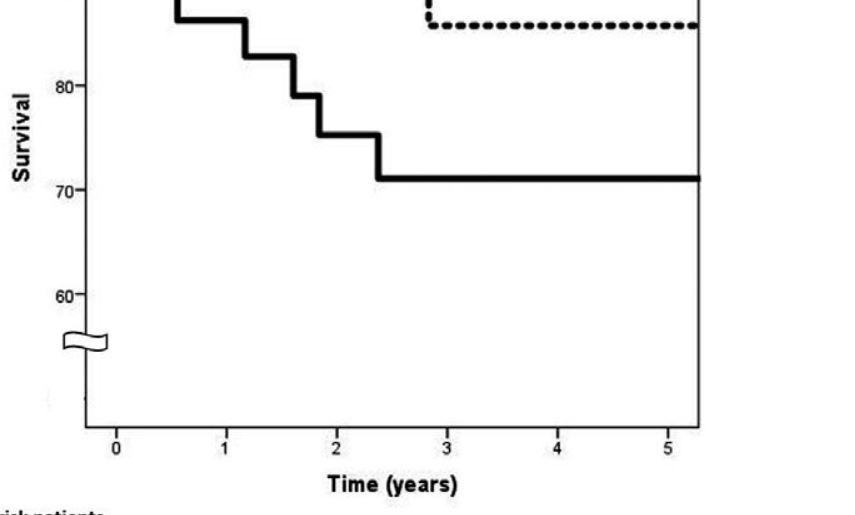

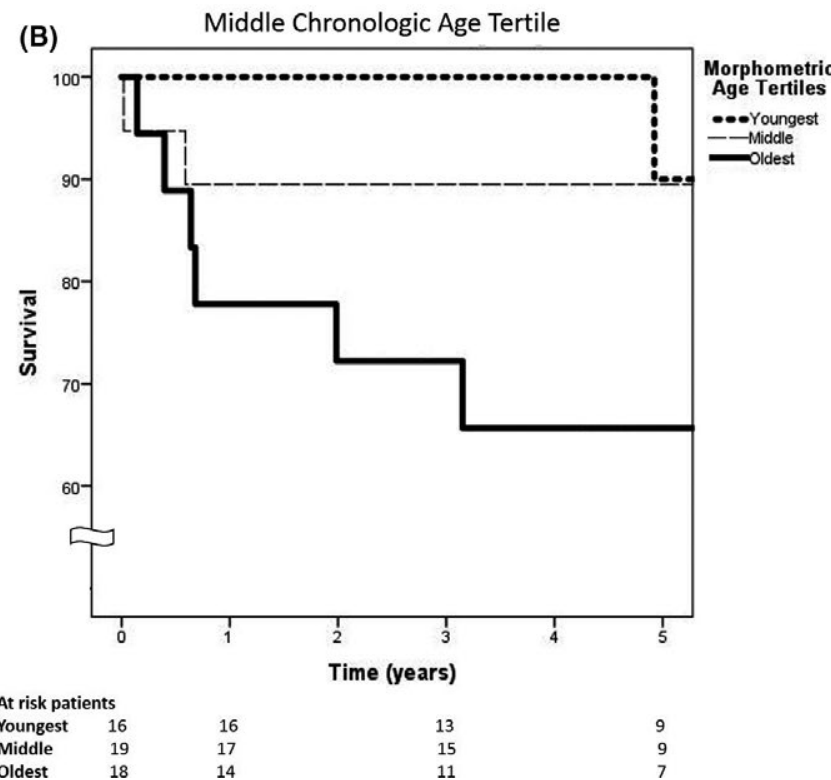

$\begin{array}{lrrrr}\text { Youngest } & 7 & 7 & 6 & 3 \\ \text { Middle } & 17 & 17 & 12 & 8 \\ \text { Oldest } & 29 & 25 & 15 & 9\end{array}$

FIGURE 3 Kaplan-Meier survival curves were stratified by morphometric age for each tertile of chronologic age. The youngest chronologic tertile (3A) includes patients with age at transplant of 19-49. The middle chronologic tertile (3B) includes patients with ages 49-61. The oldest chronologic tertile (3c) includes patients with ages 61-74

Cox regression was used to determine whether morphometric age is a significant independent predictor of survival. Morphometric age was utilized as a continuous variable in the model, as was chronologic age. All available covariates were initially evaluated univariately, and those with $P<.20$ were entered into the multivariate model. These results are summarized in Table 2 . The final subset of adjustment covariates was chosen by backwards selection, which is summarized in Table 3. The model showed that morphometric age is a significant independent predictor (hazard ratio $[\mathrm{HR}]=1.06$ per morphometric year, 95\% $\mathrm{Cl}$ : 1.03-1.08; $P<.001)$, as were diabetes mellitus ( $\mathrm{HR}=3.76,95 \% \mathrm{Cl}: 1.58-8.94 ; P=.003)$, waitlist time $(\mathrm{HR}=1.19$ per year, $95 \% \mathrm{Cl}: 1.02-1.39 ; P=.024)$, and delayed graft function $(\mathrm{HR}=2.72,95 \% \mathrm{Cl}: 1.08-6.84 ; P=0.034)$. Chronological age was not a significant covariate ( $\mathrm{HR}=1.03$ per year, $95 \% \mathrm{Cl}$ : 0.98-1.07; $P=.22$ ). All other covariates utilized in survival analysis were not significant and were excluded in the final modeling. Figure 4 utilizes the final subset of adjustment covariates to determine the adjusted survival rates for each tertile of chronologic age, further stratified by morphometric age. Using the 50th percentile of morphometric age and chronologic age, the hazard ratio can be determined for any age value. For example, the hazard ratio for a patient whose morphometric age is the 75th percentile has a $10 \%$ greater post-transplantation mortality rate than a patient with a morphometric age at the 50th percentile, covariate adjusted. Likewise, a patient with chronologic age at the 75th percentile has a $6 \%$ greater covariate-adjusted mortality rate compared to patients aged at the 50th percentile. 
TABLE 2 Univariate survival analysis

\begin{tabular}{|lll|}
\hline Covariate & $\begin{array}{l}\text { Hazard ratio } \\
(95 \% \mathrm{Cl})\end{array}$ & P value \\
\hline $\begin{array}{l}\text { Morphometric age (per morpho- } \\
\text { metric year) }\end{array}$ & $1.07(1.04-1.09)$ & $<.001$ \\
\hline ESRD: diabetes mellitus & $4.83(2.08-11.20)$ & $<.001$ \\
\hline Delayed graft function & $5.97(2.52-14.12)$ & $<.001$ \\
\hline Waitlist time (per year) & $1.22(1.08-1.38)$ & .001 \\
\hline Chronologic age (per year) & $1.05(1.02-1.09)$ & .003 \\
\hline Deceased donor & $3.07(1.36-6.92)$ & .007 \\
\hline Dialysis & $9.52(1.30-69.81)$ & .027 \\
\hline ECD & $2.47(1.03-5.91)$ & .043 \\
\hline ESRD: other & $0.49(0.22-1.09)$ & .078 \\
\hline Male gender & $1.69(0.75-3.81)$ & .20 \\
\hline Prior transplant & $0.41(0.10-1.72)$ & .22 \\
\hline Donor age & $1.02(0.99-1.05)$ & .26 \\
\hline ESRD: autoimmune & $0.32(0.04-2.34)$ & .26 \\
\hline BMI & $1.03(0.97-1.09)$ & .32 \\
\hline HCV positivity & $0.50(0.12-2.13)$ & .35 \\
\hline ESRD: HTN & $1.39(0.68-2.83)$ & .36 \\
\hline African-American race & $1.15(0.56-2.40)$ & .70 \\
\hline
\end{tabular}

TABLE 3 Results of survival analysis using Cox regression

\begin{tabular}{|lll|}
\hline Covariate & $\begin{array}{l}\text { Hazard Ratio } \\
(95 \% \mathrm{Cl})\end{array}$ & P value \\
\hline $\begin{array}{l}\text { Morphometric age* (per } \\
\text { morphometric year) }\end{array}$ & $1.06(1.03-1.08)$ & $<.001$ \\
\hline ESRD: diabetes mellitus* & $3.76(1.58-8.94)$ & .003 \\
\hline Waitlist time* (per year) & $1.19(1.02-1.39)$ & .024 \\
\hline Delayed graft function* & $2.72(1.08-6.84)$ & .034 \\
\hline Dialysis & $3.86(0.51-29.94)$ & .19 \\
\hline Chronologic age (per year) & $1.03(0.98-1.07)$ & .22 \\
\hline Deceased donor & $1.46(0.55-3.90)$ & .45 \\
\hline ESRD: other & $0.57(0.07-4.57)$ & .60 \\
\hline ECD & $1.23(0.48-3.18)$ & .67 \\
\hline
\end{tabular}

${ }^{*}$ Indicates selection to final model.

\section{4 | DISCUSSION}

We have demonstrated a novel approach to quantifying frailty as an indicator of outcomes in patients undergoing kidney transplantation. By utilizing cross-sectional imaging, we assigned a morphometric age to recipients to objectively assess mortality risk following transplantation. Our data suggest that morphometric age is a significant predictor of patient survival independent of chronologic age and comorbidity. Furthermore, among the chronologically oldest patients, those with a younger morphometric age showed increased survival compared to the patients who with older morphometric age. These results demonstrate that analytic morphomics

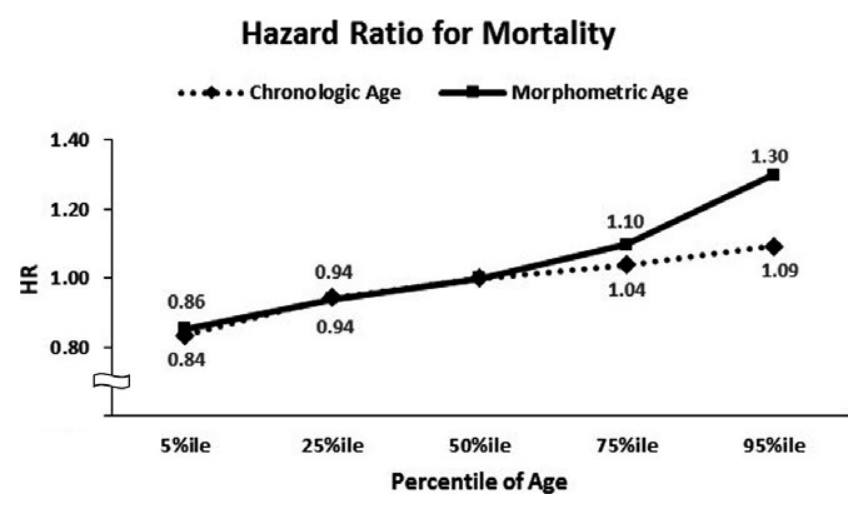

FIGURE 4 Covariate-adjusted hazard ratio for mortality was determined after selecting a subset of covariates. The reference age $(H R=1)$ was the 50 th percentile for each metric $(56.3 \mathrm{y}$ for chronologic age; 58.3 for morphometric age)

may serve as a more objective "eyeball test" of the level of patient frailty and physiological reserve in regard to fitness for kidney transplantation.

There is a growing body of literature that has investigated novel methods of preoperative risk stratification. The measure of frailty has been strongly associated with postsurgical outcomes, including mortality after kidney transplantation. ${ }^{2,9}$ Other studies have also demonstrated that sarcopenia offers a poorer prognosis compared to patients with relatively preserved muscle mass, ${ }^{18}$ even for patients only receiving conservative therapy. ${ }^{19}$ These studies often focus on indirect measures of muscle mass, such as handgrip strength or bioelectric impedance. Our study differs in that we directly measure trunk muscle size, muscle density, and vascular calcification to generate an intuitive calculation of risk, namely morphometric age. Additionally, our study suggests that morphometric age offers improved survival stratification compared to chronologic age.

This study suggests the potential for analytic morphomics to be informative for both clinicians and patients. Most importantly, the application to transplantation may help discriminate patients most likely to survive following transplantation, especially older patients. It is not uncommon in clinical parlance to describe a patient as appearing older or younger than stated age. While we observed collinearity between morphometric and chronologic age, this correlation decreases for the oldest patients, suggesting morphometric age can be viewed as a unique domain of risk assessment and serve as a proxy for the aforementioned clinical judgment. While older age was once thought to be a necessary preclusion, kidney transplantation has increasingly become a viable option for select older patients. ${ }^{20,21}$ Still, older patients are not accepted for transplantation to the same extent as younger patients, and there remain many unique challenges to transplantation in older adults. ${ }^{22-24}$ Morphometric age has the potential to improve this selection process and provide transplant surgeons more objective measures to assess and compare prospective survival benefits. Furthermore, there is a readiness to implement these strategies given that preoperative imaging has become more prevalent for transplant candidates. 
There are several important limitations of this work that must be taken into consideration. First, it is a retrospective study at a single institution with a relatively small cohort of patients-as such, it demonstrates an association, rather than causative relationship, between morphometric age and survival. Future studies should aim to include a larger sample size across multiple transplant centers, and additional work is needed to validate morphometric age in other populations. Second, patients were included in the study only if they received a preoperative abdominal CT scan within 1 year of transplant. It is conceivable that an individual patient's morphometric measures can change significantly within the course of a year, especially those with ESRD, or that the selection bias inherent in the clinical protocol indications for CT scanning could influence the results. Furthermore, the selection bias inherent in the clinical protocol indications for CT scanning could influence the results, as those who received CT scans-that is, those who were older or diabetic-are enriching the cohort with higher risk patients. Additionally, given the limitations of a retrospective study, we do not have a comprehensive set of patient comorbidities. Finally, our methodology used to determine morphometric age is based upon only three morphometric measures taken from predetermined living kidney donor and trauma patients, as these patients are intuitively the most morphometrically close to their chronological age. However, the use of this morphometric standard for comparison may not be appropriate for ESRD patients, particularly as we did not examine patients who were not eventually transplanted. Thus, our study is only applicable to ESRD patients undergoing transplantation and its implications cannot be extended to waitlist survival. Furthermore, many other methodologies and measurements could potentially inform morphometric age. Frailty may be suitable in this regard; however, no meaningful measures of frailty were apparent in the available patient records.

\section{5 | CONCLUSIONS}

Morphometric age is an independent predictor of patient survival following kidney transplantation. As patients continue to present with ESRD at older ages, additional work is warranted to assess and validate novel measures that help inform fitness for kidney transplantation. Future studies can aim to identify other morphomic factors that elucidate the complex relationship between age and physiologic reserve in the transplant candidate. It is also feasible that analytic morphomics may predict outcomes for ESRD patients who do not undergo transplantation. The potential of analytic morphomics to identify higher risk patients may also allow for intervention therapies to be implemented and improvements in organ allocation. Overall, it is conceivable that cross-sectional imaging studies will become a routine component of the standard transplant risk assessment for appropriate patients. For those centers already obtaining such scans, or for those patients who already have such imaging, morphomic assessment is easily extractable from most appropriately targeted CT scans with minimal-to-no additional cost, allowing more complete utilization of a test that has already been obtained.

\section{ACKNOWLEDGEMENTS}

None.

\section{CONFLICT OF INTEREST}

None.

\section{AUTHORS' CONTRIBUTIONS}

Michael N. Terjimanian: Performed study conception, data acquisition, data analysis, data interpretation, drafting, critical revision, and final approval. Patrick W. Underwood: Performed data analysis, data interpretation, drafting, critical revision, and final approval. David C. Cron: Performed data analysis, data interpretation, critical revision, and final approval. Joshua J. Augustine: Performed data interpretation, critical revision, and final approval. Kelly A. Noon, Devan A. Cote, Stewart C. Wang, Michael J. Englesbe: Performed data acquisition, data interpretation, critical revision, and final approval. Kenneth J. Woodside: Performed study conception, data interpretation, drafting, critical revision, and final approval. All authors agree to be accountable for all aspects of the work.

\section{REFERENCES}

1. Fried LP, Tangen CM, Walston J, et al. Frailty in older adults: evidence for a phenotype. J Gerontol A Biol Sci Med Sci. 2001;56: M146-M156.

2. Makary MA, Segev DL, Pronovost PJ, et al. Frailty as a predictor of surgical outcomes in older patients. J Am Coll Surg. 2010;210: 901-908.

3. Partridge JS, Harari D, Dhesi JK. Frailty in the older surgical patient: a review. Age Ageing. 2012;41:142-147.

4. Eisen HJ. Frailty and its impact on posttransplant outcomes: quantification of the "eyeball test." Transplantation. 2016;100:266-267.

5. Grams ME, Kucirka LM, Hanrahan CF, Montgomery RA, Massie AB, Segev DL. Candidacy for kidney transplantation of older adults. J Am Geriatr Soc. 2012;60:1-7.

6. Laging M, Kal-van Gestel JA, van de Wetering J, et al. A high comorbidity score should not be a contraindication for kidney transplantation. Transplantation. 2016;100:400-406.

7. Lai JC. Defining the threshold for too sick for transplant. Curr Opin Organ Transplant. 2016;21:127-132.

8. Lentine KL, Axelrod D, Abbott KC. Interpreting body composition in kidney transplantation: weighing candidate selection, prognostication, and interventional strategies to optimize health. Clin J Am Soc Nephrol. 2011;6:1238-1240.

9. McAdams-DeMarco MA, Law A, King E, et al. Frailty and mortality in kidney transplant recipients. Am J Transplant. 2015;15:149-154.

10. Englesbe MJ, Patel SP, He K, et al. Sarcopenia and mortality after liver transplantation. J Am Coll Surg. 2010;211:271-278.

11. Englesbe MJ, Lee JS, He K, et al. Analytic morphomics, core muscle size, and surgical outcomes. Ann Surg. 2012;256:255-261.

12. Harbaugh CM, Terjimanian MN, Lee JS, et al. Abdominal aortic calcification and surgical outcomes in patients with no known cardiovascular risk factors. Ann Surg. 2012;257:774-781.

13. Englesbe MJ, Terjimanian MN, Lee JS, et al. Morphometric age and surgical risk. J Am Coll Surg. 2013;216:976-985.

14. Waits SA, Kim EK, Terjimanian MN, et al. Morphometric age and mortality after liver transplant. JAMA Surg. 2014;149:335-340. 
15. Woodside KJ, Sung RS. Do federal regulations have an impact on kidney transplant outcomes? Adv Chronic Kidney Dis. 2016;23: 332-339.

16. Bursac Z, Gauss CH, Williams DK, Hosmer DW. Purposeful selection of variables in logistic regression. Source Code Biol Med. 2008;3:17.

17. Maldonado G, Greenland S. Simulation study of confounder-selection strategies. Am J Epidemiol. 1993;138:923-936.

18. Streja E, Molnar MZ, Kovesdy CP, et al. Associations of pretransplant weight and muscle mass with mortality in renal transplant recipients. Clin J Am Soc Nephrol. 2011;6:1463-1473.

19. Pereira RA, Cordeiro AC, Avesani CM, et al. Sarcopenia in chronic kidney disease on conservative therapy: prevalence and association with mortality. Nephrol Dial Transplant. 2015;30:1718-1725.

20. Heldal K, Hartmann A, Grootendorst DC, et al. Benefit of kidney transplantation beyond 70 years of age. Nephrol Dial Transplant. 2010;25:1680-1687.
21. McAdams-DeMarco MA, James N, Salter ML, Walston J, Segev DL. Trends in kidney transplant outcomes in older adults. J Am Geriatr Soc. 2014;62:2235-2242.

22. Knoll GA. Is kidney transplantation for everyone? The example of the older dialysis patient. Clin J Am Soc Nephrol. 2009;4:2040-2044.

23. Huang E, Segev DL, Rabb H. Kidney transplantation in the elderly. Semin Nephrol. 2009;29:621-635.

24. Knoll GA. Kidney transplantation in the older adult. Am J Kidney Dis. 2013;61:790-797.

How to cite this article: Terjimanian MN, Underwood PW, Cron DC, et al. Morphometric age and survival following kidney transplantation. Clin Transplant. 2017;31:e13066. https://doi.org/10.1111/ctr.13066 